1 NHS Providers

Cite this as: BMJ 2021;375:n2945 http://dx.doi.org/10.1136/bmj.n2945 Published: 29 November 2021

\section{As we head into a tough winter, the NHS is under huge pressure}

\section{Chris Hopson looks at the impact of ambulance handovers on patients and why everyone in the NHS needs to help rebalance the safety risk}

\section{Chris Hopson chief executive}

Over recent months, the pressures on ambulance services across the country have risen steeply, frequently making headlines. What's been particularly striking is that this pressure is happening right across the country, with all 10 ambulance trusts in England on sustained high alert.

It goes without saying that if you run a system close to, or above its capacity limits, even the smallest shifts in demand can cause major problems. You only have to look at the most recent NHS performance statistics to see that despite staff continuously going above and beyond, there are huge strains across the urgent and emergency pathway.

Ambulance services are at the sharp end, with the pressures reflected in 999 call answering, response times, and handover delays. Together, these pressures mean that the balance of risk to patient safety in the health and care system has shifted significantly, with ambulance services now bearing a disproportionate and very worrying level of risk. The scales have tipped dangerously. This is a major concern.

It poses two key questions for every trust and system leader. Where is the patient safety risk in my system greatest, and what can I and my team do to help reduce and rebalance that risk?

It's important to remember that these pressures on ambulance services are only one part of a much bigger picture. Clinicians will be acutely aware of the strain across all parts of the system-GPs, community services, mental health, hospitals, and social care. It's vital that system partners pull together-a process that will doubtless require difficult decisions and strong leadership.

What's particularly worrying is that we've not hit the traditional winter peak yet, when services typically come under even greater strain, and we are yet to find out what impact omicron, the new variant of SARS-CoV-2, will have on hospital admissions. The message from our State of the provider sector annual survey of trust leaders was loud and clear: judging by the pressure the NHS is currently under, the service is heading for the most difficult winter in its history with trusts already operating "beyond full stretch."

They face an ever-growing list of concerns including increased demand for emergency care, rising waiting lists, significant and sustained workforce shortages, potential staff burnout, the extra resource needed for vital vaccination campaigns, and the prospect of high levels of covid-19, flu, and other respiratory viruses.
Trust leaders know that it is their responsibility to support their staff to provide the best possible care to all those who need it, as rapidly and effectively as possible. That's why they are working so hard to prepare for winter and deliver vaccinations as fast as they possibly can by expanding capacity, recruiting more staff, increasing collaboration with partners across health and social care and delivering vaccinations.

But as we head into the challenging winter months, health leaders need support from the government, to tackle two immediate priorities. First, they want the government to provide emergency help to enable the social care sector to keep its existing workforce in place over the next few months. It's becoming increasingly obvious that to retain staff, the government should provide emergency support for the social care workforce, such as through a retention bonus.

Second, they want the government to give greater leadership on public health. With uncertainty about the impact that omicron may have on case rates, hospital admissions, and deaths, there is an increased need for stronger, louder, and more frequent public messaging on the continued dangers of covid-19.

Longer term, trust leaders are clear that this is a completely unsustainable position for the health and care system to be in, with real impacts on patient experiences and outcomes. So, it's imperative that we address the underlying causes affecting not just our hardworking ambulance services, but every part of our NHS: a broken workforce model, insufficient capacity to match growing demand, inadequate funding and a social care system in need of rapid, immediate support. Problems we were facing before the pandemic-made so much worse by covid-19.

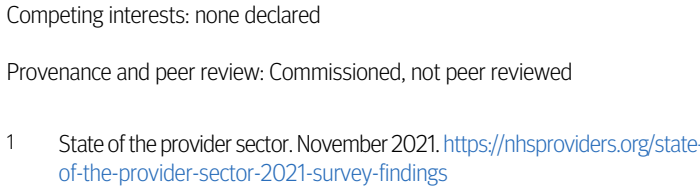

